\title{
CENTRO DE OBESIDADE INFANTIL: RELATO DE EXPERIÊNCIA
}

Larissa Soares Mariz1, Luana Batista Azevedo², Carla Campos Muniz Medeiros ${ }^{3}$, Nathalia Costa Gonzaga ${ }^{4}$, Suellen Dantas de Amorim², Larissa Camila Ferreira Souza ${ }^{5}$

RESUMO: Este estudo objetiva relatar a experiência de acadêmicos, mestrandos e professores de enfermagem no Centro de Obesidade Infantil quanto ao processo de acompanhamento e tratamento de crianças com sobrepeso e obesidade, entre agosto de 2008 a janeiro de 2012. O Centro conta com equipe multiprofissional para o acompanhamento de 349 crianças; essas têm a oportunidade de serem acompanhadas pelos profissionais, os quais interagem na discussão sobre o estado clínico e avaliam a sua evolução. A vivência no Centro propiciou perceber a real possibilidade de reunir uma equipe multidisciplinar focada em um mesmo objetivo com vistas a realizar um trabalho contínuo que possibilite meios para o alcance de resultados satisfatórios à saúde de crianças obesas ou com sobrepeso. PALAVRAS-CHAVE: Obesidade; Sobrepeso; Equipe de assistência ao paciente; Criança; Nutrição do adolescente.

\section{AN INFANTILE OBESITY CENTER: REPORT OF AN EXPERIMENT}

ABSTRACT: This study aimed to report the experience of academics, Master's degree students and professors of nursing at an Infantile Obesity Center, concerning the monitoring and treatment of overweight or obese children. The Center has a multiprofessional team for the monitoring of 349 children; these have the opportunity to be monitored by the healthcare professionals, who interact in discussion about the child's clinical state and evaluate its evolution. Experience in the Center made it possible to see what happens when a multidisciplinary team is united, focussed on the same objective, with the aim of carrying out a continuous work which ensures means for the achievement of satisfactory results with the health of overweight or obese children.

KEYWORDS: Obesity; Overweight; Patient care team; Child; Adolescent nutrition.

\section{CENTRO DE OBESIDAD INFANTIL: RELATO DE EXPERIENCIA}

RESUMEN: Este estudio tuvo el objetivo de relatar la experiencia de académicos, mestrandos y profesores de enfermería en el Centro de Obesidad Infantil cuanto al proceso de acompañamiento y tratamiento de niños con sobrepeso y obesidad, entre agosto de 2008 y enero de 2012. El Centro cuenta con equipo multiprofesional para el acompañamiento de 349 niños; eses tienen la oportunidad de ser acompañados por los profesionales, los cuales interactúan en la discusión acerca del estado clínico y evalúan su evolución. La vivencia en el Centro propició percibir la real posibilidad de reunir un equipo multidisciplinar alrededor de un mismo objetivo con vistas a realizar un trabajo continuo que posibilite medios para llegar a resultados satisfactorios a la salud de niños obesos o con sobrepeso.

PALABRAS CLAVE: Obesidad; Sobrepeso; Equipo de asistencia al paciente; Niño; Nutrición del adolescente.

${ }^{1}$ Enfermeira. Mestranda pelo Programa de Pós-Graduação em Enfermagem da Universidade Estadual da Paraíba - UEPB.

${ }^{2}$ Acadêmica do Curso de Graduação em Enfermagem, Extensão e Iniciciação Científica pela UEPB.

${ }^{3}$ Médica. Doutora em Saúde da Criança e do Adolescente. Professora dos Programas de Pós-Graduação em Saúde Pública da Universidade Federal da Paraíba e do Programa de Pós-Graduação em Enfermagem da UEPB.

${ }^{4}$ Enfermeira. Mestranda em Saúde Pública pelo Programa de Pós-Graduação da UEPB.

${ }^{5}$ Acadêmica do Curso de Graduação em Enfermagem, Extensão pela UEPB. 


\section{INTRODUÇÃO}

A obesidade se tornou um grave problema de saúde pública, apresentando-se como uma verdadeira epidemia mundial ${ }^{(1)}$, incluindo a faixa etária infantojuvenil, o que acarreta o aparecimento de alterações cardiovasculares e metabólicas em idade precoce ${ }^{(2-3)}$. Ao reconhecer o excesso de peso como um fator de risco para diversas enfermidades ${ }^{(4)}$, há a necessidade de incluir, nas rotinas dos serviços de saúde da atenção básica, a abordagem nutricional como uma prática efetiva e cotidiana, alcançando assistência integral e humanizada ao paciente com excesso de peso ${ }^{(5)}$.

Uma vez instalada a obesidade infantil, o caráter multifatorial da doença demanda intervenção interdisciplinar em seu tratamento, o que inclui a participação de equipe multiprofissional para realizar estratégias de atuação terapêutica, proporcionando abordagem integral do paciente ${ }^{(6)}$. Buscando-se intervir junto às crianças e adolescentes obesos de Campina Grande - Paraíba, profissionais de saúde, professores e acadêmicos da Universidade Estadual da Paraíba instituíram o Centro de Obesidade Infantil (COI), a fim de acompanhar crianças e adolescentes obesos, ou com sobrepeso, em seu tratamento e promover melhora na qualidade de vida, no momento atual e futuro. Neste contexto, chama-se atenção ao estado de saúde e não somente ao aspecto físico, bem como, a necessidade de incluir familiares e acompanhantes como coautores, no processo de preservação e recuperação da saúde.

Este estudo relata a experiência vivida no COI por acadêmicos, mestrandos e professores de enfermagem quanto ao processo de acompanhamento e tratamento de crianças com sobrepeso e obesidade entre agosto de 2008 a janeiro de 2012.

\section{ASPECTOS METODOLÓGICOS DA EXPERI- ENCIA}

\section{Delineando o funcionamento do COI}

O COI está implantado no Instituto de Saúde Elpídio de Almeida, Campina Grande-PB, desde agosto de 2008. Este município situa-se no centro do Estado da Paraíba, liderando política e geograficamente outras 60 cidades ao seu redor. Possui cerca de 500 mil habitantes, sendo a segunda cidade mais populosa do estado, e conta com 92 equipes de saúde da família, 9 núcleos de apoio a saúde da família; 19 hospitais, distribuídos entre públicos - federal, estadual e municipais - filan- trópicos e privados. O Centro busca atender crianças e adolescentes entre dois e 18 anos, encaminhados pelas Unidades Básicas de Saúde da Família e Secretaria Municipal de Saúde, que se enquadram no estado nutricional de sobrepeso ou obesidade.

É fato que existe uma complexidade da reformulação dos hábitos alimentares, e a necessidade da construção de estratégias em conjunto com o paciente, a fim de que esse seja capaz de compreender a necessidade e a importância do problema. Por esse motivo, o COI tem por objetivo possibilitar melhora da autoestima, por meio do estímulo a adotar um estilo de vida saudável para toda a família, prevenção das co-morbidades da obesidade e manutenção ou perda de peso.

O serviço conta com a participação de pesquisadores e equipe multiprofissional, composta por endocrinologistas, nutricionistas, psicóloga, enfermeiro, assistente social e preparador físico. $\mathrm{O}$ atendimento segue a seguinte rotina: acadêmicos de enfermagem e farmácia executam a triagem inicial, por meio de um questionário sobre o estado de saúde, desde o nascimento, e realizam antropometria (peso, estatura, circunferência abdominal e pressão arterial). Em seguida, o paciente é atendido pela assistente social, que aborda os aspectos socioeconômicos em consulta única. Ao serviço de endocrinologia compete a avaliação do estado clínico geral, a solicitação dos exames laboratoriais e da bioimpedância, além das orientações básicas sobre a alimentação e prática de atividade física. Onutricionista avalia os hábitos alimentares e prescreve as mudanças necessárias, e o preparador físico disponibiliza um programa de atividade física. Alguns casos específicos também são encaminhados para a psicóloga, como pacientes que relatam ser ansiosos ou impulsivos. Em todas as consultas os pais ou acompanhantes fazem-se presentes, visto a necessidade de orientação de conduta conjunta.

Nos momentos que antecedem o atendimento ambulatorial, a enfermagem desenvolve atividades lúdico-pedagógicas; temas relacionados à obesidade são abordados de forma atraente aos participantes. Este é um espaço destinado não somente para a propagação do saber, mas também para promover um ambiente para testemunhos de sucesso no tratamento, assim como, esclarecimento de dúvidas e dificuldades enfrentadas pelos pacientes.

O tratamento no COI tem duração indeterminada, buscando-se a mudança no estilo de vida e um índice de massa corpórea (IMC) adequado, segundo as seguintes categorias: obesidade acentuada (IMC $\geq$ percentil 97), obesidade (percentil $95 \geq$ IMC $<97)$, sobrepeso $(85 \geq$ IMC $<95)$ e eutrófico $(10>\text { IMC }<85)^{(7)}$. 
Atualmente são atendidos regularmente cerca de 350 pacientes e aproximadamente 300 aguardam em uma lista de espera a desistência ou surgimento de novas vagas, a fim de iniciarem o tratamento. Apesar do bom número de unidades básicas e centros de saúde no município, o COI é o único centro de referência para acompanhamento e tratamento à crianças e adolescentes com excesso de peso, assim como para o desenvolvimento de novas pesquisas na área.

\section{Experiências vivenciadas}

Há muitas experiências a serem destacadas desde o período de planejamento do Centro. Inicialmente, para a implantação do COI, foi primordial a aprovação de um projeto pela Fundação de Apoio à Pesquisa da Paraíba (FAPESQ), possibilitando o financiamento de parte do material utilizado pela equipe: esfigmomanômetro de mercúrio e manguitos de tamanhos adequados para a faixa etária infanto-juvenil, aparelho de bioimpedância para medição do percentual de gordura, fita métrica inelástica com precisão de $0,1 \mathrm{~cm}$, balança digital do tipo plataforma, com capacidade para $150 \mathrm{~kg}$ e precisão de $0,1 \mathrm{~kg}$, e estadiômetro com precisão de $0,1 \mathrm{~cm}$.

Após a aquisição do material, para a divulgação, a Secretária de Saúde do Município enviou uma carta a todas as unidades com Estratégia Saúde da Família, solicitando o encaminhamento de crianças e adolescentes entre dois e 18 anos para o acompanhamento no Centro. Foi marcado um encontro com o objetivo de treinar médicos e enfermeiros das unidades de saúde para a avaliação do estado nutricional, porém foram poucos os que compareceram. Desta forma, muitos profissionais de saúde da atenção primária não treinados realizam o diagnóstico visual do sobrepeso/ obesidade, o que resulta no encaminhamento de alguns casos falsos-positivos ao COI.

Nessa trajetória de acompanhamento de crianças e adolescentes obesos é possível elencar potencialidades e fragilidades do COI. Como limitações, destacam-se: impossibilidade de aumentar o número de vagas devido à inserção de poucos profissionais, resultando em longa lista de espera; infraestrutura física insuficiente para a realização de atividade físicas grupais e necessidade de melhorias nas salas de atendimento e no ambiente de espera; equipe de enfermagem não treinada para desempenhar seu papel no tratamento de obesos; e longo período de tratamento, visto que o paciente recebe alta ao atingir a classificação eutrófica e, infelizmente, poucos têm conseguido.
A frequência de muitos pacientes aos atendimentos não é contínua. Os pais e/ou responsáveis apresentam algumas das seguintes justificativas: falta de tempo para levar os filhos até o COI; dificuldade financeira para pagamento de transporte, apesar do COI localizarse no centro do município, o que facilita o acesso da população; muitos pacientes hesitam em continuar o tratamento, enquanto que os seus pais assumem a condução destes ao COI, acarretando faltas em alguns atendimentos. Desta forma, com o objetivo de evitar a descontinuidade do tratamento, a equipe realiza contatos telefônicos para os pais, convidando-os a retornar aos atendimentos - em muitos casos, esta estratégia tem sido eficaz.

Em contrapartida, são diversos os fatores positivos que têm sido observados, a destacar: acompanhamento multidisciplinar; manutenção de parcerias importantes (Universidade Estadual da Paraíba, Universidade Federal de Pernambuco, Prefeitura e Unidades de Saúde da Família); realização de exames laboratoriais e de imagem de alta complexidade sem ônus para os pacientes; desenvolvimento de atividades de educação em saúde; participação da família no tratamento; e redução do IMC e dos valores lipídicos; e o desenvolvimento de pesquisas atuais e inovadoras sobre a temática.

Os pacientes inseridos no tratamento são acompanhados pelos profissionais, que interagem na discussão sobre seu estado clínico e avaliam a sua evolução. A cada consulta o paciente recebe esclarecimentos sobre a prática de exercícios físicos e dieta. Este se constitui em um espaço aberto para o esclarecimento das dúvidas e explicação de como substituir a cultura alimentar da família por novos costumes, de forma a conduzir a criança ou adolescente a uma nutrição adequada para cada faixa etária. Também neste momento, os pais dialogam sobre os seus sentimentos e as crianças têm a oportunidade de escolher, acordado com a dieta, suas preferências alimentares.

O envolvimento da família tem sido fundamental para a mudança do estilo de vida dos pacientes. As pessoas dessa faixa etária seguem padrões paternos; se esses não forem modificados ou manejados em conjunto, um insucesso do tratamento já é previsto ${ }^{(5)}$.

Por esse motivo, além das crianças e adolescentes, a família também participa das atividades de educação em saúde realizadas na sala de espera do serviço. $\mathrm{Na}$ perspectiva dos profissionais, as ações educativas têm surtido efeito positivo, uma vez que pacientes e familiares demonstram compreender aspectos associados à obesidade. Além disto, como resultado dos eventos 
ocorridos no ambulatório, é possível perceber a interação dos profissionais com as crianças, fator importante para que haja melhor eficácia ao tratamento, visto que metas rígidas de perda de peso podem não ser alcançadas, gerando frustrações e perda de motivação ${ }^{(9)}$.

Outras atividades são promovidas em datas comemorativas, como na Páscoa, Dia das Crianças e Natal. São momentos recreativos com incentivo à prática de exercícios físicos e, após, é oferecido um lanche saudável para os participantes. Dessa maneira, os pacientes e familiares percebem que é possível praticar atividade física "brincando" e adquirir uma alimentação saudável sem maiores transtornos.

Por meio do acompanhamento multidisciplinar, o COI tornou-se um campo para o desenvolvimento de pesquisas em saúde, incluindo projetos financiados pela FAPESQ e pela Universidade Estadual da Paraíba, trabalhos de extensão e iniciação científica, dissertações de mestrado e publicação de artigos em revistas nacionais, abordando temas discutidos no cenário nacional como: síndrome metabólica, resistência insulínica, acantose nigrans, pressão arterial elevada, dislipidemia, doença cardiometabólica, entre outros.

\section{CONSIDERAÇÕES FINAIS}

O Centro constitui um ambiente onde crianças e adolescentes, bem como os seus familiares, podem ser assistidos, contam com a disponibilidade de recursos de saúde para tratamento e acompanhamento do excesso de peso, a fim de minimizar os riscos à saúde. Entende-se a importância da implantação de centros de tratamento da obesidade em outras cidades do Brasil, visto que a prevalência de crianças e adolescentes com esse perfil nutricional aumenta anualmente.

O COI, por meio de sua dinâmica, ressalta a viabilidade de reunir uma equipe multidisciplinar, focada no mesmo objetivo, para realizar um trabalho contínuo que possibilite meios para que a clientela alcance efeitos satisfatórios. No entanto, faz-se necessário a capacitação de novos profissionais para trabalhar com o público-alvo com vistas a reduzir o número de pacientes em lista de espera por atendimento. Por meio do atendimento multidisciplinar e das atividades de educação em saúde foi possível estabelecer com o paciente e sua família, maior contato e, possivelmente, maior vínculo e gerando melhores resultados. Inspirados nessa experiência outros centros para tratamento de obesidade infantojuvenil podem ser implantados no Brasil.

\section{REFERENCIAS:}

1. WHO - World Health Organization. Obesity and overweight: Global strategy on diet, physical activity and healthy. Geneva, 2009 [acesso em 16 nov 2010]. Disponível: www.who.int/dietphysicalactivity/media/ en/gsfs_obesity.pdf

2. Carvalho DF, Paiva AZ, Melo ASO, Ramos AT, Medeiros JS, Medeiros CCM, et al. Perfil lipídico e estado nutricional de adolescentes. Rev. bras. epidemiol. 2007;10(4): 491-8.

3. Dias ABFA. Fatores associados ao sobrepeso e obesidade em adolescentes de 10 a 14 anos atendidos no posto de atenção infantil (PAI) em Balneário Camboriú - SC [dissertação]. Itajaí (SC): Universidade Vale do Itajaí; 2007.

4. Faustino EB, Azevedo EB, Silva PC, Sales S, Barros W. Diabetes melittus: busca ativa em portadores de obesidade. Cogitare enferm. 2011;16(1): 110-15.

5. Ministério da Saúde (BR). Secretaria de Atenção à Saúde. Departamento de Atenção Básica. Obesidade. Cadernos de Atenção Básica, n. 12 Série A. Normas e Manuais Técnicos. Brasília; 2006.

6. Gastaldon B, Martins JC, Poltroniéri KV. Obesidade infantil: um problema do presente com olhares para o futuro - promovendo o ser família saudável no quotidiano junto à enfermagem. Florianópolis; 2007.

7. CDC. Table for calculated body mass index values for selected heights and weights for ages 2 to 20 years. Developed by the National Center for Health Statistic in collaboration with the National Center for Chronic Disease Prevention and Health Promotion; 2000. Publicado em maio de 2002, modificado 20/04/2001. 